\title{
Seleção de genótipos de arroz tolerantes à salinidade durante a fase vegetativa
}

\author{
Selection of genotypes of salinity tolerant rice during the vegetative phase
}

\author{
Palmira Cabral Sales de Melo ${ }^{1}$ Clodoaldo José da Anunciação Filho ${ }^{2}$ Francisco José de Oliveira ${ }^{2 *}$ \\ Gerson Quirino Bastos ${ }^{2}$ José Nildo Tabosa ${ }^{1}$ Venézio Felipe dos Santos ${ }^{1}$ \\ Maria Rita Cabral Sales de Melo ${ }^{3}$
}

\begin{abstract}
RESUMO
O objetivo deste trabalho foi avaliar o grau de tolerância $e$ sensibilidade à salinidade de genótipos de arroz durante a fase vegetativa da planta. $O$ experimento foi conduzido sob condições de telado, nas dependências da Empresa Pernambucana de Pesquisa Agropecuária-IPA (Recife-PE), em 1996. Foram avaliados doze genótipos de arroz, sendo dez tolerantes e dois sensíveis à salinidade no estádio de desenvolvimento vegetativo. $O$ delineamento experimental foi em blocos ao acaso com arranjo fatorial (doze genótipos $x$ quatro níveis de $\mathrm{NaCl}$ ), em três repetições. Os resultados constataram existência de variabilidade entre os genótipos de arroz na população estudada para tolerância e sensibilidade à salinidade. As linhagens PR492, PR504, CNA8250, CNA8262 e CNA8267 são tolerantes e a CNA8270, CNA8258, CNA8269, PR475 e PR477 são sensíveis à salinidade dos solos durante a fase vegetativa.
\end{abstract}

Palavras-chave: Oryza sativa, cultivar, solos salinos, condutividade elétrica, caracteres fenotípicos.

\section{ABSTRACT}

The objective of this work was to evaluate the degree of tolerance and sensibility to the salinity of genoty,pes of rice (Oryza sativa L.) during the vegetative phase of the plant. The experiment was lead under greenhouse conditions at IPA (Empresa Pernambucana de Pesquisa Agropecuária), in 1996. Twelve genotypes of rice had been evaluated, being ten tolerant and two sensible ones to salinity in the vegetative development stadium. The randomized blocks design were performed into three repetitions of factorials arrangements (twelve genotypes $x$ four levels of $\mathrm{NaCl}$ ). Results had evidenced variability existence among the genotypes of rice, in the specific population, for tolerance and sensitivity to the salinity. Samples PR492, PR504, CNA8250, CNA8262 and CNA8267 can be considered tolerant to salinity, while the genotypes CNA8270, CNA8258, CNA8269, PR475 and PR477 are sensible to salinity of soil during the vegetative phase.

Key words: Oryza sativa, cultivar, saline soils, electrical conductivity, phenotypic characters.

\section{INTRODUÇÃO}

Os solos salinos ocupam cerca de $20 \%$ das áreas irrigadas do semi-árido do Nordeste Brasileiro. Além disso, as áreas de baixada ribeirinha concentram, na época seca do ano, grande quantidade de sais solúveis, o que limita o cultivo da maioria das culturas. Estas áreas que apresentam umidade no solo podem permitir o cultivo de genótipos de arroz tolerantes à salinidade (PRISCO \& AGUIAR, 1987). BLACK (1975) afirma que a salinidade pode afetar as plantas de três maneiras: a) redução na disponibilidade hídrica, já que os sais solúveis presentes em excesso nos solos salinos aumentam a retenção dos solutos na água do solo, reduzindo dessa forma a água disponível para as plantas; b) o efeito específico dos íons $\mathrm{Cl}^{-}$e $\mathrm{Na}^{+}$podem estimular ou inibir as reações metabólicas e ter um efeito tóxico na célula vegetal; e c) redução no transporte de solutos devido à presença de certos íons que podem

${ }^{1}$ Empresa Pernambucana de Pesquisa Agropecuária (IPA), Av. Gal. San Martin, 1371. Bonji, Caixa Postal 1022, CEP: 50761-000, Recife, PE, Brasil. E-mail: palmiracabral@globo.com

${ }^{2}$ Departamento de Agronomia da UFRPE, Rua D. Manoel de Medeiros, s/n - Dois Irmãos. CEP: 52.171-900, Recife, PE, Brasil. e-mail: franseol@uol.com.br (autor para correspondência).

${ }^{3}$ Departamento de Biologia da UFRPE. Recife, PE, Brasil. 
inibir ou estimular a absorção de outros íons pelas plantas. As respostas das plantas podem ser atribuídas a vários mecanismos específicos, como capacidade em excluir o sal dos tecidos; capacidade de realizar um completo ajustamento osmótico e estabilidade das membranas, macromoléculas e sistemas enzimáticos (GALE, 1975).

A obtenção de um estande adequado de plantas em áreas salinas é freqüentemente difícil, em função do decréscimo na porcentagem de germinação (OSTER et al., 1984). Uma das alternativas para contornar esse problema é a seleção de genótipos mais tolerantes à salinidade nos estádios de germinação e estabelecimento da plântula. Na literatura, já existem estudos a respeito do efeito da salinidade nos diferentes estádios de desenvolvimento de plantas de arroz (CAMPOS \& ASSUNÇÃO, 1990; SALIM et al., 1990; AHMED \& GUPTA, 1991; YAN \& TAN, 1991, YEO et al. 1991; LUTTS et al., 1996), havendo, no entanto, a necessidade de outras pesquisas que visem à identificação de genótipos tolerantes à salinidade. Em cultivos de arroz efetuados em solos com condutividade elétrica entre 1,4 e 25,6dS m ${ }^{-1}$, GORE \& BHAGWAT (1988) verificaram uma redução na altura de planta, produção de perfilhos e produtividade. Quarenta cultivares de arroz avaliados em diferentes níveis de salinidade por FAGERIA (1991) indicaram uma diferença altamente significativa entre elas para altura de planta, perfilho e produção de matéria seca da plântula. Estudos de AKITA \& CABUSLAY (1990), sob uma CE de $10 \mathrm{dS} \mathrm{m}{ }^{-1}$, em cultivares de arroz, observaram reduções de $13 \%, 15 \%$ e $27 \%$, respectivamente, para altura de planta, número de perfilhos e produção de matéria seca de plântula, quando comparados com a testemunha. As cultivares avaliadas diferiram significativamente nas suas respostas quanto ao crescimento sob salinidade. Estes autores afirmaram que a maioria dos materiais com pouca tolerância, em condições altamente salinas, podem ser eliminados como critério de seleção e propõem que se eleve potencialmente os níveis de salinidade dos experimentos.

O objetivo deste trabalho foi avaliar o grau de tolerância à salinidade de genótipos de arroz na fase vegetativa.

\section{MATERIAL E MÉTODOS}

O experimento foi conduzido sob condições de telado, nas dependências da Empresa Pernambucana de Pesquisa Agropecuária-IPA (Recife-PE). Foram avaliados doze genótipos de arroz (Oryza sativa L.), sendo dez tolerantes (PR475, PR477, PR492, PR504,
CNA8258, CNA8264, CNA8266, CNA8267, CNA8269e CNA8270) e dois sensíveis (CNA8250 e CNA8262) no estádio de germinação. O delineamento experimental usado foi o de blocos ao acaso em arranjo fatorial (12 genótipos x 4 níveis de $\mathrm{NaCl}$ ), com três repetições. Foi utilizado como substrato solo Aluvião, o qual depois de seco ao ar, destorroado e passado em peneira de $2 \mathrm{~mm}$, foi colocado em 144 vasos de plástico com capacidade para 12 litros, perfurado ao lado próximo à base. Os vasos receberam na base, uma camada de brita, forrada com tecido de algodão a fim de facilitar a drenagem. Sobre esta, foi colocado o solo (g/vaso) previamente homogeneizado com a quantidade de $\mathrm{NaCl}$ correspondente a cada tratamento. As quantidades de $\mathrm{NaCl}$ utilizadas para cada tratamento foram determinadas a partir da condutividade elétrica calculada através da equação $\mathrm{y}=1,617476+4,7102764 \mathrm{x}$, ou seja, $\mathrm{NaCl} \mathrm{g} \mathrm{kg}{ }^{-1}$ solo $(0 ; 0,92 ; 2,20 ; 3,48), \mathrm{NaCl} g$ vaso $^{-1}(0 ; 9,2 ; 22,0 ; 34,8)$ e condutividade elétrica calculada $\left(0 ; 6 ; 12\right.$ e $\left.18 \mathrm{dS} \mathrm{m}^{-1}\right)$. Cada vaso, ao receber $10 \mathrm{~kg}$ de solo homogeneizado com ou sem o $\mathrm{NaCl}$, foi irrigado com água de qualidade, classificada como $\mathrm{C}_{2} \mathrm{~S}_{2}$ (RICHARDS, 1974). Nestas condições, o sistema permaneceu por um período de 30 dias, objetivando maior segurança, quanto à estabilidade da incubação. Após o período de incubação, cada vaso foi sobreposto em outro vazio e sem perfuração (vaso coletor), objetivando receber o excesso de água. Após este período, fez-se análise do extrato saturado do solo no Laboratório de Química do IPA e, para isso, retirou-se $300 \mathrm{~g}$ de solo de cada recipiente. O experimento permaneceu até aos 53 dias após semeado, com 10 sementes. No $12^{\circ}$ dia após semeio, foi feito o transplantio entre repetições de acordo com o tratamento, quando necessário. No $21^{\circ}$ dia, fez-se o desbaste deixando-se 4 plantas vaso ${ }^{-1}$. Só foi necessária irrigação até o semeio, sendo o restante conduzido no período das chuvas que manteve o solo sob condições de campo ou saturação. $\mathrm{O}$ excesso de água foi drenado naturalmente para o vaso coletor e sempre que possível, retornado ao solo do vaso de onde havia drenado.

Foram avaliadas as seguintes variáveis: a) condutividade elétrica do extrato saturado (CE) - obtida no Laboratório de Química do IPA, através de um condutivímetro, modelo E 389, utilizando-se amostra de $300 \mathrm{~g}$ de solo/vaso; b) altura de planta (AP) - obtida pela mensuração a partir do nível do solo até o ápice da folha mais alta de forma distendida no $17^{\circ}$ e $53^{\circ}$ dia após semeio; c) nota para vigor (NV) - foram atribuídas, utilizando-se escala preconizada pelo Manual de Métodos de Pesquisa em Arroz (EMBRAPA, 1977), assim discriminadas: Nota 1- plantas extra vigorosas, Nota 3 - plantas vigorosas, Nota 5 - plantas com vigor 
normal, Nota 7- plantas menos vigorosas que o normal, Nota 9 - plantas fracas e doentes ou mortas; d) número de perfilho (NP) - foi obtido através de contagem numérica dos perfilhos antes da colheita, no 52일 após semeio; e) área foliar (AF) - realizou-se a coleta do material no 53 dia após semeio, utilizando -se uma lâmina para fazer o corte das plantas rente ao solo. Essas foram pesadas e, após a separação das folhas, foi realizada a mensuração da área foliar com o auxílio de um "medidor de área foliar" computadorizado modelo LI $3000 \mathrm{~A}$; f) produção de matéria seca (PMS) imediatamente, após a colheita da parte aérea conforme descrito para determinação da área foliar, sendo pesada e posta em estufa de circulação de ar, sob temperatura de $65^{\circ} \mathrm{C}$ até peso constante. Após esta etapa, o material foi novamente pesado e registrada a massa da matéria seca; g) percentagem de matéria seca (\%MS) - obtida através do quociente entre peso seco e peso verde do material colhido e expressa em percentagem; $h$ ) taxa de crescimento absoluto (TCA) - obtida a partir da altura de planta, considerando as mensurações realizadas no $17^{\circ}$ e $53^{\circ}$ dia após semeio, conforme BLEASDALE (1977). Os dados relativos ao número de perfilhos foram transformados em $\sqrt{x+5}$ para se processar a análise estatística. Os dados referentes a nota para vigor (atribuídas subjetivamente mediante escala de 1 a 9) e stand final, também sofreram transformação através de gg $\sqrt{x+1}$ antes de serem analisados, de acordo com GOMES (1990). Procedeu-se a análise de variância para testar as fontes de variação e suas interações. Com forma de desdobrar os graus de liberdade da interação genótipos x doses, efetuou-se a análise de regressão, e comparação de médias através do teste SCOTT \& KNOTT(1974).

\section{RESULTADOS E DISCUSSÃO}

Os resultados do teste $\mathrm{F}$ detectaram diferenças entre os genótipos e níveis de salinidade para os caracteres altura da planta no 53ㅇdia, número de perfilhos, área foliar, produção e percentagem de matéria seca. As variáveis condutividade elétrica do extrato saturado do solo e nota de vigor no $17^{\circ}$ e $27^{\circ}$ dia apresentaram diferenças apenas para salinidade. A nota de vigor no $27^{\circ}$ dia e taxa de crescimento absoluto foram significativas $(\mathrm{P}<0,05)$ apenas para genótipo (Tabela 1).

A altura média de plantas (AP) aos 53 dias após semeio (Tabela 1), diferiram pelo teste Scott-Knott. Os materiais PR492, CNA8250, CNA8266 e CNA8267 exibiram as maiores alturas neste estágio de desenvolvimento. Resultados similares foram encontrados por SAXENA \& PANDEY (1981), GORE \& BHAGWAT (1988), PUNYAWARDENA \&
DHAMASRI (1989), SALIM et al. (1990), YAN \& TAN (1991), YEO et al. (1991) e FAGERIA (1991). Estudos realizados por MARASSI et al. (1989), em 81 cultivares e nove linhagens locais de arroz para tolerância à salinidade, mostraram que os materiais eram mais tolerantes na fase reprodutiva que na vegetativa e que a altura de planta e comprimento da panícula correlacionaram-se fortemente com a salinidade a $8 \mathrm{dS} \mathrm{m}^{-1}$. Todos os genótipos apresentaram comportamento de crescimento linear através da função $\mathrm{Y}=-0,5147 \mathrm{x}+$ 66,25 e $\mathrm{R}^{2}=0,9493$ (Figura 1A). O coeficiente de regressão $b=-0,5147$ é significativo $(P<0,05)$, o que indica que à medida que se aumenta a inclusão da condutividade elétrica (CE) na saturação do solo, a altura das plantas reduz-se à razão de $0,5147 \mathrm{~cm}$ para cada unidade de acréscimo da C.E aplicada.

Para os valores de condutividade elétrica do extrato saturado do solo (C.E), não houve diferença. Este resultado expressa a precisão e o ajuste da função para o intervalo entre zero e $18 \mathrm{dS} \mathrm{m}^{-1}$ de condutividade elétrica (Figura 1B). Nestas circunstâncias, os genótipos foram semeados com a equivalência entre os níveis propostos de salinidade $\left(0,6,12\right.$ e $\left.18 \mathrm{dS} \mathrm{m} \mathrm{m}^{-1}\right) \mathrm{e}$ a condutividade elétrica real obtida no condutivímetro que foram 4,4; 9,6; 16,7 e 23,3dS m ${ }^{-1}$. Com relação às médias de nota para vigor (NV) no 17ํㅡ e 27을 dia após semeio (Tabela 1), os genótipos diferiram pelo teste Scott-Knott. Os genótipos PR492, PR504, CNA8250, CNA8262 e CNA8267, com as menores notas médias no $17^{\circ}$ e no $27^{\circ}$ dia, refletiram o desenvolvimento equivalente à plantas vigorosas, sofrendo interferência mínima dos efeitos da salinidade nos níveis submetidos. Os resultados indicam que à medida que aumenta a condutividade elétrica (CE) na saturação do solo, a nota para vigor nos períodos estudados, também aumenta à razão de 0,19 para cada unidade de acréscimo da CE aplicada (Figuras 1C e 1D).

Quanto ao número de perfilhos (NP) os genótipos PR504, CNA8250 e CNA8262 apresentaram o mesmo comportamento, mas diferiram dos demais. No entanto, o genótipo CNA8262 exibiu o maior número de perfilhos, em média, 4,57, enquanto o CNA8270, o menor número de perfilhos, em média de 3,37. Entretanto, o CNA8262, PR504 e CNA8250 exibiram maior número de perfilhos quando comparados com os demais, indicando serem mais tolerantes ao $\mathrm{NaCl}$. O efeito da salinidade sob o perfilhamento apresentou comportamento linear mostrando que, à medida que aumenta o nível de salinidade, o número de perfilhos diminui (Figura 1E). Segundo PEDROSO (1989), uma das características associada a alto rendimento de grão é o perfilhamento vigoroso, pois viabiliza a produção de um elevado número de panículas. A queda na produção de perfilhos com o aumento da salinidade do 
Tabela 1 - Valores médios obtidos em 12 genótipos de arroz e resultados da análise de variância para as variáveis condutividade elétrica do extrato saturado do solo (C.E), altura da planta (AP) dos genótipos de arroz no $53^{\circ}$ dia após a semeadura, nota para vigor (NV) no $17^{0}$ e $27^{\circ}$ dia após a semeadura, número de perfilhos (NP), área foliar (AF), produção de matéria seca (PMS), percentagem de matéria seca (\%MS) e taxa de crescimento absoluto (TCA), em função dos níveis de condutividade elétrica do extrato saturado do solo. Recife (PE), 1996.

\begin{tabular}{|c|c|c|c|c|c|c|c|c|c|}
\hline \multirow{2}{*}{ Genótipos $^{1}$} & \multirow{2}{*}{$\begin{array}{c}\text { C.E } \\
\mathrm{dS} \mathrm{m} \mathrm{m}^{-1}\end{array}$} & \multirow{2}{*}{$\frac{\mathrm{AP}}{53^{0}}$} & \multicolumn{2}{|c|}{ NV } & \multirow{2}{*}{ NP } & \multirow{2}{*}{$\begin{array}{c}\mathrm{AF} \\
\left(\mathrm{cm}^{2}\right)\end{array}$} & \multirow{2}{*}{$\begin{array}{l}\text { PMS } \\
(\mathrm{g})\end{array}$} & \multirow{2}{*}{$\% \mathrm{MS}$} & \multirow{2}{*}{$\begin{array}{l}\text { TCA } \\
(\%)\end{array}$} \\
\hline & & & $17^{0}$ & $27^{0}$ & & & & & \\
\hline PR475 & $13,1 \mathrm{a}$ & $57,9 \mathrm{~b}$ & $4,0 \mathrm{a}$ & $2,0 \mathrm{~b}$ & $3,67 b$ & $5,03 \mathrm{~b}$ & $9,34 \mathrm{a}$ & $24,3 b$ & $0,82 b$ \\
\hline PR477 & $14,4 \mathrm{a}$ & $57,1 \mathrm{~b}$ & $4,5 \mathrm{a}$ & $2,4 a$ & $3,97 \mathrm{~b}$ & $5,82 \mathrm{~b}$ & $9,55 \mathrm{a}$ & $25,9 a$ & $0,92 \mathrm{a}$ \\
\hline PR492 & $12,6 \mathrm{a}$ & $62,3 a$ & $3,5 b$ & $2,0 \mathrm{~b}$ & $3,85 b$ & $6,72 \mathrm{a}$ & $11,34 \mathrm{a}$ & $26,6 a$ & $0,91 \mathrm{a}$ \\
\hline PR504 & $12,8 \mathrm{a}$ & $53,6 \mathrm{~b}$ & $3,5 b$ & $2,2 b$ & $4,50 \mathrm{a}$ & $5,95 b$ & $10,13 \mathrm{a}$ & $28,7 \mathrm{a}$ & $0,81 b$ \\
\hline CNA8250 & $13,6 \mathrm{a}$ & $65,4 \mathrm{a}$ & $3,5 b$ & $2,2 b$ & $4,18 \mathrm{a}$ & $6,14 b$ & $9,74 \mathrm{a}$ & $26,7 \mathrm{a}$ & $1,01 \mathrm{a}$ \\
\hline CNA8258 & $13,4 \mathrm{a}$ & $51,3 b$ & $5,4 \mathrm{a}$ & $2,3 a$ & $3,70 \mathrm{~b}$ & $4,49 \mathrm{~b}$ & $6,54 b$ & $23,9 b$ & $0,75 b$ \\
\hline CNA8262 & $14,6 \mathrm{a}$ & $58,7 \mathrm{~b}$ & $3,2 b$ & $2,1 b$ & $4,57 \mathrm{a}$ & $8,13 \mathrm{a}$ & $10,46 a$ & $26,8 \mathrm{a}$ & $0,84 \mathrm{~b}$ \\
\hline CNA8264 & $13,6 \mathrm{a}$ & $60,1 b$ & $4,6 a$ & $2,4 a$ & $3,80 \mathrm{~b}$ & $5,60 \mathrm{~b}$ & $7,66 b$ & $22,8 b$ & $0,86 b$ \\
\hline CNA8266 & $12,6 \mathrm{a}$ & $63,8 \mathrm{a}$ & $4,2 \mathrm{a}$ & $2,3 a$ & $3,82 b$ & $5,66 \mathrm{~b}$ & $7,14 \mathrm{~b}$ & $23,5 b$ & $0,99 a$ \\
\hline CNA8267 & $13,5 \mathrm{a}$ & $65,5 \mathrm{a}$ & $3,2 b$ & $2,1 b$ & $3,66 b$ & $5,41 \mathrm{~b}$ & $7,70 \mathrm{~b}$ & $26,8 \mathrm{a}$ & $0,96 \mathrm{a}$ \\
\hline CNA8269 & $13,6 \mathrm{a}$ & $56,6 \mathrm{~b}$ & $4,2 \mathrm{a}$ & $2,2 b$ & $3,71 \mathrm{~b}$ & $5,03 \mathrm{~b}$ & $7,39 b$ & $20,9 b$ & $0,71 b$ \\
\hline CNA8270 & $13,1 \mathrm{a}$ & $59,1 \mathrm{~b}$ & $4,6 a$ & $2,4 a$ & $3,37 \mathrm{~b}$ & $4,53 b$ & $6,24 b$ & $23,3 b$ & $0,96 \mathrm{a}$ \\
\hline C.V $(\%)$ & 15,0 & 16,3 & 15,9 & 14,3 & 19,1 & 33,2 & 34,6 & 19,96 & 27,4 \\
\hline DMS (5\%) & 3,04 & 13,16 & 0,55 & 0,43 & 1,01 & 2,58 & 4,07 & 6,82 & 0,33 \\
\hline F(salinidade) & $592,56^{* *}$ & $7,35^{* *}$ & $39,10^{* *}$ & $40,17^{* *}$ & $20,55^{* *}$ & $19,10^{* *}$ & $35,04^{* *}$ & $10,22^{* *}$ & $0,18^{\mathrm{ns}}$ \\
\hline F(genótipo) & $1,14^{\mathrm{ns}}$ & $2,54^{* *}$ & $1,57^{\mathrm{ns}}$ & $2,05^{*}$ & $2,72^{* *}$ & $3,37^{* *}$ & $3,81^{* *}$ & $2,30^{* *}$ & $1,93^{*}$ \\
\hline Transformação & & & & $\sqrt{x+1}$ & $\sqrt{x+0,5}$ & & & & \\
\hline
\end{tabular}

${ }^{1}$ Médias seguidas por letras distintas diferem entre si ao nível de 5\% de probabilidade, pelo teste de SCOTT \& KNOTT (1974).

solo coincide com as observadas por diversos autores (SAXENA \& PANDEY, 1981; FAGERIA et al., 1981; GORE \& BHAGWAT, 1988 e FAGERIA, 1991).

Os valores médios de área foliar entre os genótipos diferiram. Os genótipos PR492 e CNA8262 não diferem entre si, mas diferiram estatisticamente dos demais genótipos e exibiram as maiores áreas foliares (Tabela 1). A análise de regressão apresentou configuração linear, ou seja, quando aumenta a condutividade elétrica (CE) na saturação do solo, reduz a área foliar das plantas explicada pela figura $1 \mathrm{~F}$.

Os resultados para a produção de matéria seca mostram que os genótipos PR475, PR477, PR492 e CNA8262, não diferiram, mas foram diferentes entre as demais linhagens e exibiram os valores mais altos para este caráter (Tabela 1). As linhagens PR492, CNA8262 e PR504 destacaram-se com as maiores produções de matéria seca, respectivamente, 11,34g, 10,46g e 10,13g; e com o menor valor a CNA8270. FAGERIA et al. (1981) concluíram que as cultivares de arroz classificadas como tolerantes, produziram significativamente mais matéria seca do que as sensíveis. Para a matéria seca a análise de regressão apresentou comportamento linear, conforme ilustrado na figura $1 \mathrm{G}$. Para a percentagem de matéria seca os genótipos PR477, PR492, PR504, CNA8250, CNA8262 e CNA8267 não houve diferença entre si, mas diferiram dos genótipos PR475, CNA8258, CNA8264, CNA8266, CNA8269 e CNA8270. A relação entre a percentagem de matéria seca e a condutividade elétrica explica que, quando aumentou a condutividade elétrica, a percentagem de matéria seca decresceu à razão de 0,3023\% para cada unidade de acréscimo da C.E (figura 1H). As linhagens PR492, PR504, CNA8250, CNA8266, CNA8267 e CNA8270 exibiram as maiores taxas de crescimento absoluto. A taxa de crescimento absoluto representa a velocidade média de crescimento ao longo do período de observação.

Na tabela 2, a análise de variância para altura da planta no $17^{\circ}$ dia e nota para vigor no $53^{\circ}$ dia, apresentou diferenças para interação genótipo x níveis de salinidade. A altura da planta no $17^{\circ}$ dia dos genótipos de arroz diferiu para os níveis de salinidade 9,4 e 16,7dS m ${ }^{-1}$. A linhagem CNA8269 diferiu estatisticamente das PR477 e CNA8270 para o nível de 


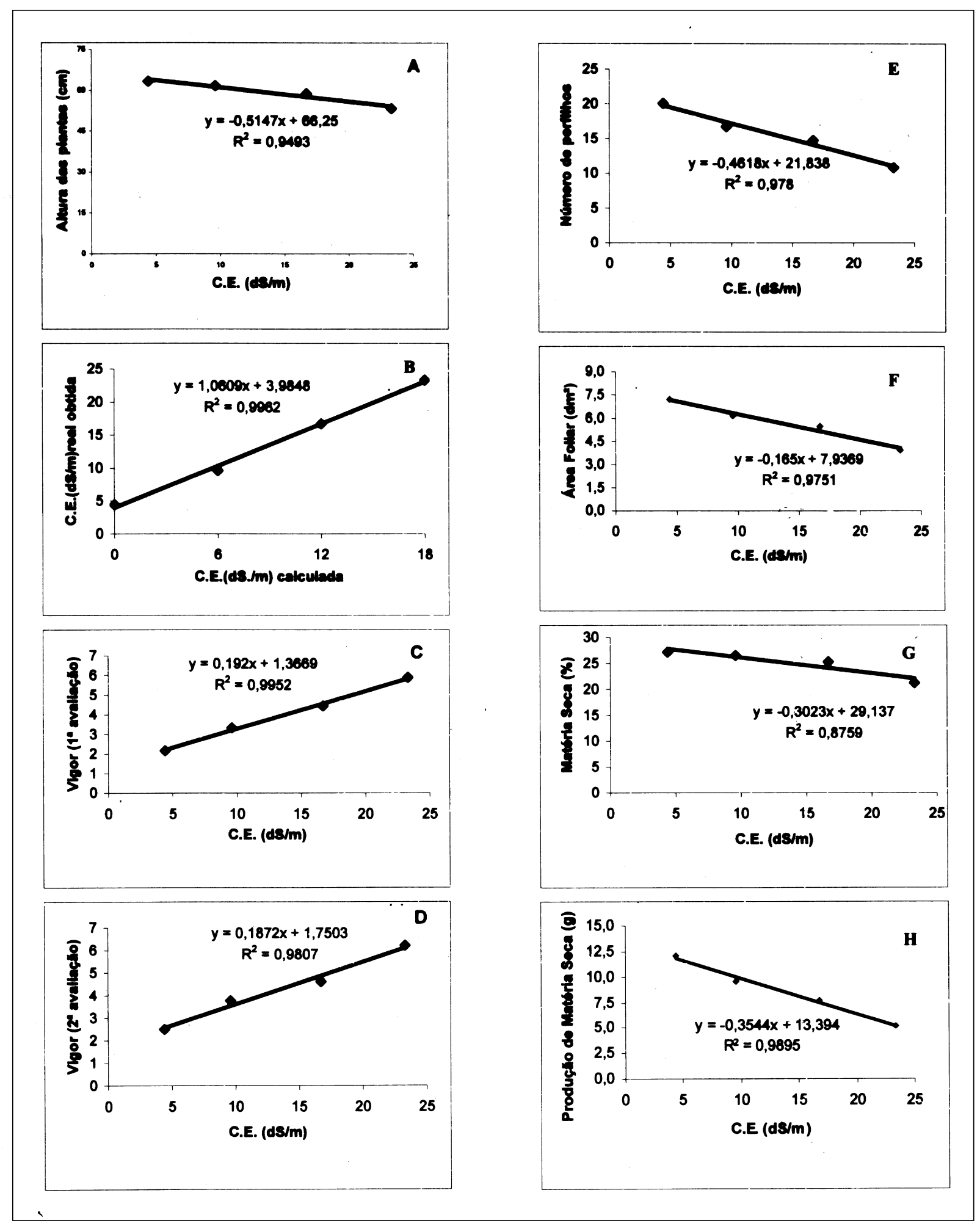

Figura 1- Condutividade elétrica versos: A - altura das plantas no $53^{\circ}$ dias após semeio ; B - Condutividade real obtida após 30 dias de irrigação; C - Vigor no $17^{\circ}$ dia após semeio; D - Vigor no $27^{\circ}$ dia após semeio; $\mathbf{E}$ - $\mathrm{N}^{\circ}$ de perfilhos; $\mathbf{F}$ - Área foliar; $\mathbf{G}$ Produção de matéria seca e $\mathbf{H}$ - Percentagem de matéria seca.

Ciência Rural, v.36, n.1, jan-fev, 2006. 
Tabela 2 - Valores médios obtidos em 12 genótipos de arroz para altura da planta no $17^{\circ}$ dia após a semeadura, nota para vigor no $53^{\circ}$ dia após a semeadura e número de perfilhos, em função dos níveis de condutividade elétrica do extrato saturado do solo $\left(\mathrm{dSm}{ }^{-1}\right)$. Recife (PE), 1996.

\begin{tabular}{|c|c|c|c|c|c|c|c|c|c|c|c|c|}
\hline \multirow{3}{*}{ Genótipo $^{1}$} & \multicolumn{4}{|c|}{ Altura da planta $(\mathrm{cm})$} & \multicolumn{4}{|c|}{ Nota para vigor } & \multicolumn{4}{|c|}{ Stand final } \\
\hline & \multicolumn{4}{|c|}{ Níveis de condutividade elétrica } & \multicolumn{4}{|c|}{ Níveis de condutividade elétrica } & \multicolumn{4}{|c|}{ Níveis de condutividade elétrica } \\
\hline & 4,4 & 9,6 & 16,7 & 23,3 & 4,4 & 9,6 & 16,7 & 23,3 & 4,4 & 9,6 & 16,7 & 23,3 \\
\hline PR475 & $32,4 a$ & $34,1 \mathrm{ab}$ & $26,5 \mathrm{ab}$ & $21,7 \mathrm{a}$ & $1,6 a$ & $1,0 \mathrm{~b}$ & $3,7 \mathrm{ab}$ & $5,7 \mathrm{ab}$ & $2,23 \mathrm{a}$ & $2,23 a$ & $2,23 \mathrm{a}$ & $1,82 \mathrm{abc}$ \\
\hline PR477 & $31,1 \mathrm{a}$ & $22,8 b c$ & $23,8 \mathrm{ab}$ & $19,0 \mathrm{a}$ & $1,6 \mathrm{a}$ & $4,3 \mathrm{ab}$ & $5,0 \mathrm{ab}$ & $5,7 \mathrm{ab}$ & $2,23 \mathrm{a}$ & $2,23 a$ & $2,23 a$ & $2,23 \mathrm{a}$ \\
\hline PR492 & $35,0 \mathrm{a}$ & $30,2 \mathrm{abc}$ & $26,8 \mathrm{ab}$ & $26,7 \mathrm{a}$ & $1,0 \mathrm{a}$ & $1,7 \mathrm{ab}$ & $3,0 \mathrm{ab}$ & $3,0 \mathrm{~b}$ & $2,23 \mathrm{a}$ & $2,23 a$ & $2,15 \mathrm{a}$ & $2,15 \mathrm{ab}$ \\
\hline PR504 & $28,0 \mathrm{a}$ & $25,3 \mathrm{abc}$ & $22,7 \mathrm{ab}$ & $22,1 \mathrm{a}$ & $2,3 a$ & $4,3 \mathrm{a}$ & $4,3 \mathrm{ab}$ & $3,7 \mathrm{ab}$ & $2,23 \mathrm{a}$ & $2,23 \mathrm{a}$ & $2,23 \mathrm{a}$ & $1,96 \mathrm{ab}$ \\
\hline CNA 8250 & $31,0 \mathrm{a}$ & $32,6 \mathrm{ab}$ & $29,6 \mathrm{ab}$ & $22,6 a$ & $1,6 \mathrm{a}$ & $1,7 \mathrm{ab}$ & $3,0 \mathrm{ab}$ & $3,7 \mathrm{ab}$ & $2,23 \mathrm{a}$ & $2,23 \mathrm{a}$ & $2,23 \mathrm{a}$ & $2,23 \mathrm{a}$ \\
\hline CNA8258 & $30,9 a$ & $26,5 \mathrm{abc}$ & $17,2 \mathrm{~b}$ & $23,4 a$ & $3,0 \mathrm{a}$ & $5,0 \mathrm{a}$ & $6,3 \mathrm{a}$ & $5,0 \mathrm{ab}$ & $2,23 \mathrm{a}$ & $2,23 \mathrm{a}$ & $1,65 \mathrm{a}$ & $2,23 \mathrm{a}$ \\
\hline CNA8262 & $32,2 \mathrm{a}$ & $30,6 a b c$ & $29,1 \mathrm{ab}$ & $21,3 \mathrm{a}$ & $2,3 a$ & $1,7 \mathrm{ab}$ & $2,3 b$ & $5,7 \mathrm{ab}$ & $2,23 \mathrm{a}$ & $2,23 \mathrm{a}$ & $2,23 \mathrm{a}$ & $2,23 \mathrm{a}$ \\
\hline CNA8264 & $35,9 a$ & $30,7 \mathrm{ab}$ & $32,1 \mathrm{a}$ & $18,3 \mathrm{a}$ & $2,3 a$ & $3,0 \mathrm{ab}$ & $4,3 \mathrm{ab}$ & $7,0 \mathrm{ab}$ & $2,23 \mathrm{a}$ & $2,23 \mathrm{a}$ & $1,98 \mathrm{a}$ & $1,55 b c$ \\
\hline CNA8266 & $33,9 \mathrm{a}$ & $33,4 a b$ & $27,7 \mathrm{ab}$ & $17,7 \mathrm{a}$ & $1,0 \mathrm{a}$ & 2,3ab & $3,7 \mathrm{ab}$ & $5,7 \mathrm{ab}$ & $2,23 \mathrm{a}$ & $2,23 \mathrm{a}$ & $1,98 \mathrm{a}$ & $1,62 \mathrm{abc}$ \\
\hline CNA8267 & $31,5 \mathrm{a}$ & $32,1 \mathrm{ab}$ & $31,5 \mathrm{ab}$ & $28,0 \mathrm{a}$ & $1,7 \mathrm{a}$ & $2,3 \mathrm{ab}$ & $3,7 \mathrm{ab}$ & $3,7 \mathrm{ab}$ & $2,23 \mathrm{a}$ & $2,23 \mathrm{a}$ & $2,15 \mathrm{a}$ & $1,88 \mathrm{ab}$ \\
\hline CNA8269 & $37,2 \mathrm{a}$ & $35,0 \mathrm{a}$ & $33,1 \mathrm{a}$ & $18,4 \mathrm{a}$ & $1,7 \mathrm{a}$ & $1,0 \mathrm{~b}$ & $3,0 \mathrm{ab}$ & $7,7 \mathrm{a}$ & $2,23 \mathrm{a}$ & $2,23 \mathrm{a}$ & $2,15 \mathrm{a}$ & $1,24 \mathrm{c}$ \\
\hline \multirow[t]{5}{*}{ CNA8270 } & $31,1 \mathrm{a}$ & $19,2 \mathrm{c}$ & $28,2 \mathrm{ab}$ & $20,2 \mathrm{a}$ & $2,3 a$ & $3,7 \mathrm{ab}$ & 4,3ab & $7,7 \mathrm{a}$ & $2,23 \mathrm{a}$ & $2,23 \mathrm{a}$ & $1,96 \mathrm{a}$ & $1,65 \mathrm{abc}$ \\
\hline & \multicolumn{4}{|c|}{$\mathrm{CV}(\%)=15,7$} & \multicolumn{4}{|c|}{$\mathrm{CV}(\%)=15,4$} & \multicolumn{4}{|c|}{$\mathrm{CV}(\%)=10,97$} \\
\hline & \multicolumn{4}{|c|}{$\mathrm{F}($ gen $x$ sal $)=1,70^{*}$. } & \multicolumn{4}{|c|}{$\mathrm{F}($ gen $x s a l)=1,61^{*}$. } & \multicolumn{4}{|c|}{$\mathrm{F}($ gen $x$ sal $)=1,97^{\text {*k. }}$. } \\
\hline & \multicolumn{4}{|c|}{$\operatorname{DMS}(5 \%)=11,94$} & \multicolumn{4}{|c|}{$\operatorname{DMS}(5 \%)=0,86$} & \multicolumn{4}{|c|}{$\operatorname{DMS}(5 \%)=0,64$} \\
\hline & & & & & & & & & Transfom & $\sqrt{x+1}$ & & \\
\hline
\end{tabular}

${ }^{1}$ Médias seguidas por letras distintas diferem entre si ao nível de 5\% de probabilidade, pelo teste de SCOTT \& KNOTT (1974).

salinidade de $9,4 \mathrm{dS} \mathrm{m}^{-1}$. Entretanto, no nível de salinidade de $16,7 \mathrm{dS} \mathrm{m}^{-1}$, as linhagens CNA8269 e CNA8258 diferiram entre si, a primeira exibiu altura média superior aos demais materiais, nos níveis de salinidade de 4,4, e 9,4dS m ${ }^{-1}$. A linhagem CNA8264 atingiu a maior altura quando submetidos os níveis de 9,6 e 16,7 dS m${ }^{-1}$, tendo refletido interferência mínima dos efeitos de salinidade nestes níveis. Dentre os genótipos avaliados, o que apresentou a maior variação para altura neste estádio de desenvolvimento, em função dos níveis de salinidade, foi o CNA8269. No entanto, o mais estável foi o CNA8267 que apresentou a menor variação, evidenciando pouca influência do $\mathrm{NaCl}$, quando comparado com os demais, podendo ser considerado como o mais tolerante ao $\mathrm{NaCl}$ em relação à altura de planta no $17^{\circ}$ dia. Análise de regressão foi linear para todos os genótipos avaliados. Verifica-se que os valores médios de nota para vigor dos genótipos de arroz aos 53 dia após semeio, diferiram apenas entre as linhagens CNA8258 e CNA8262, no nível de salinidade de $16,7 \mathrm{dS} \mathrm{m}^{-1}$. Ao nível de condutividade elétrica de 23,3dS m${ }^{-1}$ a linhagem PR492 foi diferente da CNA8269 e CNA8270. Resultados semelhantes foram obtidos por CAMPOS \& ASSUNÇÃO (1990) no estádio de desenvolvimento vegetativo da planta. Os genótipos CNA8267, CNA8250, PR504 e PR492 apresentaram as menores notas para vigor no maior nível de salinidade $\left(23,3 \mathrm{dS} \mathrm{m} \mathrm{m}^{-1}\right)$ indicando menor sensibilidade ao $\mathrm{NaCl}$. Entretanto, o genótipo CNA8258 apresentou valores altos de notas para vigor no $53^{\circ}$ dia em todos os níveis de salinidade, evidenciando maior sensibilidade na presença de $\mathrm{NaCl}$ neste estádio de desenvolvimento. O CNA8270, CNA8269, CNA8264, CNA8262, PR475 e PR477 exibiram os maiores valores de notas para vigor no $53^{\circ}$ no nível de $23,3 \mathrm{dS} \mathrm{m}^{-1}$ e consequientemente, maior sensibilidade ao $\mathrm{NaCl}$.

O "stand" final, não apresentou diferença entre genótipos e níveis de salinidade nos níveis de 4,4, 9,6 e 16,7. No entanto, no nível mais alto $\left(23,3 \mathrm{dS} \mathrm{m}^{-1}\right)$ os genótipos PR477, CNA8250, CNA8258 e CNA8262 não diferiram entre si, mas estas linhagens diferiram das linhagens CNA8264, CNA8266 e CNA8269. No nível máximo de salinidade, as linhagens PR477, CNA8250, CNA8258 e CNA8262 permaneceram com o "stand" inalterado e na linhagem CNA8269 ocorreu uma redução drástica.

\section{CONCLUSÕES}

Os genótipos de arroz na população estudada apresentam variabilidade para tolerância e 
sensibilidade à salinidade. As linhagens PR492, PR504, CNA8250, CNA8262 e CNA8267 são tolerantes e a CNA8270, CNA8258, CNA8269, PR475 e PR477 são sensíveis à salinidade dos solos durante a fase vegetativa do arroz.

\section{APRESENTAÇÃO}

Parte da dissertação de mestrado apresentada pelo primeiro autor à Universidade Federal Rural de Pernambuco (UFRPE), Recife, PE, Brasil.

\section{REFERÊNCIAS}

AHMED, J.; GUPTA, S. Germination and growth of some saltresistant selections in high salt concentration solutions. International Rice Research Newsletter, Manila, v.16, n.5, p.15, 1991 .

AKITA, S.; CABUSLAY, G.S. Physiological basis of differential response to sanility in rice cultivars. Plant and Soil, Dordrecht, v.123, p.227-294, 1990.

BLACK, C.A. Relaciones suelo-planta. Buenos Aires: Hemisferio Sur, 1975. V.1.

BLEASDALE, J.K.A. Fisiologia vegetal. São Paulo: USP, 1977. $176 \mathrm{p}$.

CAMPOS, I.S.; ASSUNÇÃO, M.V. Efeitos do cloreto de sódio na germinação e vigor de plântulas de arroz. Pesquisa Agropecuária Brasileira, Brasília, v.25, n.6, p.837-843, 1990.

EMBRAPA, Centro Nacional de Pesquisa de Arroz e Feijão (Goiânia, GO). Manual de métodos de pesquisa em arroz: primeira aproximação. Goiânia: EMBRAPA - CNPAF, 1977. $106 \mathrm{p}$.

FAGERIA, N.K. Tolerance of rice cultivars to salinity. Pesquisa Agropecuária Brasileira, Brasília, v.26, n.2, p.281288, 1991.

FAGERIA, N.K. et al. Avaliação de cultivares de arroz para tolerância à salinidade. Pesquisa Agropecuária Brasileira, Brasília, v.16, n.5, p.667-681, 1981.

GALE, J. Water balance and gas exchange of plants under saline condition. In: POLJAKOFF-MAYBER, A; GALE, J. (Ed). Plants in saline environments. Berlin: SpringerVeriag, 1975. p.168-185.

GOMES, F.P. Curso de estatística experimental. 12.ed. São Paulo: Nobel, 1990. 468p.
GORE, S.R.; BHAGWAT, K.A. Performance of rice cultivar Mashuri at different salinity levels. International Rice Research Newsletter, Manila, v.13, n.6, p.21, 1988.

LUTTS, S. et al. Effects of salt stress on growth, mineral nutrition and proline accumalation in relation to osmotic adjustment in rice (Oryza sativa L.) cultivar dilheing in salinity resistence. Plant growth regulation, Springer-Verlag New York, v.19, p.207-218, 1996.

MARASSI, J.E. et al. Performance of selected rice genotypes in alkaline, saline, and normal soils and their interaction witch climate factores. International Rice Research Newsletter, Manila v.14, n.6, p.10-11, 1989.

OSTER, J.D. et al. Mangment alternatives: crop, water and soil. California Agriculture, Oakland, v.36, p.29-32, 1984.

PEDROSO, B.A. Introdução / Melhoramento. In: Arroz irrigado: obtenção de cultivares. Porto Alegre: SAGRA, 1989 . p.8. / p.34-68.

PRISCO, J.T.; AGUIAR, P.A.A. Pesquisas fitotécnicas para as áreas irrigadas com problemas de sais no Nordeste. Fortaleza: Minter/Sudene, 1987. 17p.

PUNYAWARDENA, B.V.R.; DHARMASRI, L.C. Effect of salinity on rice germination and seedling growth. International Rice Research Newsletter, Manila, v.14, n.5, p.18, 1989 .

RICHARDS, L.A. Diagnóstico y rehabilitaction de suelos salinos y sódicos. México: Limusa, 1974. p.1-6. Origen y natureza de suelos salinos y sódicos; p.75-88: La calidad del agua para riego; p.59-73: Respuesta de las plantas y selección de cultivos para suelos salinos y sódicos.

SALIM, M. et al. Salinity stress and varietal resistance in rice: effects on white backed plant hopper. Crop Science, Madison, v.30, p.654-659, 1990.

SAXENA, H.K.; PANDEY, U.K. Physiological studies on salt tolerance of ten rice varieties. I Growth and yield aspects. Indian Journal of Plant Pathology, London, v.24, n.1, p.6168, 1981

SCOTT, A. J.; KNOTT, M. A. A cluster analysis method for grouping means in the analysis of variance. Biometrics, Raleigh, v.30, n.3, p.507-512, 1974.

YAN, X.; TAN, K. Screening rice varieties for SALT tolerance in the greenhouse. International Rice Research Newsletter, Manila, v.16, n.1, p.16-17, 1991.

YEO, A.R. et al. Short-and long-term effects of salinity on leaf growth in rice (Oryza sativa L.). Journal of Experimental Botany, Oxford, v.42, n.240, p.881-889, 1991. 